Pacific Journal of Mathematics

SCHLICHT TAYLOR SERIES WHOSE CONVERGENCE ON
THE UNIT CIRCLE IS UNIFORM BUT NOT ABSOLUTE paul ERdôs, F. Hertzog and G. Pika 


\title{
SCHLICHT TAYLOR SERIES WHOSE CONVERGENCE ON THE UNIT CIRCLE IS UNIFORM BUT NOT ABSOLUTE
}

\author{
P. Erdös, F. Herzog, and G. Piranian
}

1. Summary. That a Taylor series which converges uniformly on the unit circle $C$ need not converge absolutely on $C$ was proved by Hardy [2] (see also Landau [4, p.68]; for a simpler example, see Herzog and Piranian [3, Section 4]). The present paper exhibits two functions that are schlicht on the closed unit disc, and whose Taylor series converge uniformly but not absolutely on C. Each of the examples satisfies an additional restrictive requirement: the first function has only one singular point on $C$, and the Taylor series

$$
\sum_{k=0}^{\infty} a_{k} z^{m} k
$$

of the second function has the property that $\lim \left(m_{k+1}-m_{k}\right)=\infty$.

The condition that (1) represent a schlicht function and converge uniformly but not absolutely on $C$ imposes restrictions on the sequence of exponents $\left\{m_{k}\right\}$. For the condition implies that $\sum_{k=0}^{\infty} m_{k}\left|a_{k}\right|^{2}<\infty$ (see Landau [4, p.65]); since, by Schwarz's inequality, we have

$$
\left(\sum_{k=0}^{\infty}\left|a_{k}\right|\right)^{2} \leq \sum_{k=0}^{\infty} m_{k}\left|a_{k}\right|^{2} \cdot \sum_{k=0}^{\infty} 1 / m_{k},
$$

it follows that

$$
\sum_{k=0}^{\infty} 1 / m_{k}=\infty
$$

It remains an open question whether the condition implies a restriction on $\left\{m_{k}\right\}$ which is stronger than (2). 
In the construction of both examples, the basic idea consists of the observation that if

$$
h(z) \equiv z+k \omega\left[1-(1-z / \omega)^{1 / n}\right]
$$

(where $k$ is a real constant, $|\omega|=1, n$ is a positive integer and the function $(1-z / \omega)^{1 / n}$ is chosen to be positive when $\left.z=\omega / 2\right)$, then $h(z)$ maps the unit disc into a region which consists roughly of the unit disc with a tooth of length $k$ protruding at the point $z=\omega$. The tooth can be made arbitrarily narrow by choosing $n$ large enough. If additional terms are joined to the right member of (3), the map of the unit disc by $h(z)$ bristles with teeth; and if the lengths, widths and positions of these teeth are chosen appropriately, the Taylor series of $h(z)$ converges uniformly, but not absolutely, on $C$. The geometric and analytic motivation for the devices that induce $h(z)$ to satisfy the additional requirements will be obvious from the text.

2. The first example. Let $\left\{\phi_{j}\right\}$ be a decreasing sequence of real numbers $\left(2 \pi>\phi_{1}, \phi_{j} \rightarrow 0\right)$, and $\left\{\delta_{j}\right\}$ a sequence of positive numbers such that the discs $\left|z-e^{i \phi_{j}}\right|<\delta_{j}$ are disjoint. For each index $j, \Omega_{j}$ shall denote the complement, relative to the $\operatorname{disc}|z|<2$, of the union of the $\operatorname{disc}\left|z-e^{i \phi_{j}}\right|<\delta_{j}$ and the line segment $z=r e^{i \phi}, 1<r<2$. Also, for each index $j, \rho_{j}$ shall denote a real number subject to the condition

$$
1<\rho_{j}<1+\delta_{j} / 2
$$

$N_{j}$ shall denote a positive integer such that, for every $\rho_{j}$ satisfying (4) and every $n_{j}$ greater than $N_{j}$, the function

$$
f_{j}(z) \equiv 1-\left(1-z / \omega_{j}\right)^{1 / n_{j}}
$$

$\left(\omega_{j}=\rho_{j} e^{i \phi_{j}},\left(1-z / \omega_{j}\right)^{1 / n_{j}}\right.$ positive when $\left.z=\omega_{j} / 2\right)$ satisfies the inequality $\left|f_{j}(z)\right|<2^{-j}$ throughout $\Omega_{j}$.

We now proceed to select the integers $n_{j}$ in such a way that, in a suitable region about the origin, the series

$$
z+\sum_{j=1}^{\infty} \omega_{j} f_{j}(z) / j
$$


converges uniformly to a function which is endowed with the desired properties. To this end, we define the numbers $a_{m, j}$ by the equations

$$
f_{j}(z)=\sum_{m=1}^{\infty} a_{m, j}\left(z e^{-i \phi_{j}}\right)^{m}
$$

keeping in mind that at this stage of the discussion the numbers $a_{m, j}$ must be regarded as functions of the still undetermined constants $\rho_{j}$ and $n_{j}$. It should be noted that the $a_{m, j}$ are all positive; that, for each fixed $j$, they form a decreasing ' sequence whose first element is $\left(\rho_{j} n_{j}\right)^{-1}$; and that

$$
\sum_{m=1}^{\infty} a_{m, j}=f_{j}\left(e^{i \phi_{j}}\right)=1-\left(1-1 / \rho_{j}\right)^{1 / n_{j}} .
$$

Let $n_{1}$ be an integer greater than $N_{1}$; and let $\rho_{1}$ be a real number satisfying condition (4), and near enough to one so that $\left(1-1 / \rho_{1}\right)^{1 / n_{1}}<2^{-1}$. Once $n_{\nu}$ and $\rho_{\nu}$ have been chosen for $\nu=1,2, \cdots, j-1$, let $M_{j}$ denote an integer so large that

$$
\sum_{\nu=1}^{j-1} \sum_{m>M} a_{m}, \nu<2^{-j}
$$

and let $n_{j}$ be greater than $N_{j}$ and so large that, for all $\rho_{j}$ satisfying (4),

$$
\sum_{m \leq M} a_{m}, j<2^{-j}
$$

finally, let $\rho_{j}$ be chosen near enough to one so that

$$
\left(1-1 / \rho_{j}\right)^{1 / n_{j}}<2^{-j} .
$$

Then the series (5) converges uniformly in some closed region whose interior contains all points of the closed unit disc except the point $z=1$. Its sum $F(z)$ is therefore continuous on the closed disc, and holomorphic at all its points except at $z=1$. The Taylor series $\sum_{m=1}^{\infty} a_{m} z^{m}$ of $F(z)$ does not converge absolutely on $C$; for

$$
a_{m}=\sum_{\nu=1}^{\infty}\left(\omega_{\nu} / \nu\right) a_{m, \nu} e^{-i \phi_{\nu m}}, \quad m \geq 2,
$$


and therefore

$$
\left|a_{m}\right| \geq a_{m, j} / j-2 \sum_{\nu \neq j} a_{m, \nu}
$$

hence it follows from (6)-(9) that

$$
\sum_{M_{j}<m \leq M_{j+1}}\left|a_{m}\right| \geq j^{-1}-O\left(2^{-j}\right)
$$

That $\sum_{m=1}^{\infty} a_{m} z^{m}$ converges uniformly on $C$ can be shown directly; but it will also follow from the continuity of $F(z)$ and Fejér's Theorem, once univalence has been established (see Fejér [1] or Landau [4, pp. 65, 66]).

To establish univalence of the function $F(z)$, it is sufficient to note that

$$
(d / d z)\left[-\omega_{j}\left(1-z / \omega_{j}\right)^{1 / n_{j}}\right]=\left(1 / n_{j}\right)\left(1-z / \omega_{j}\right)^{1 / n_{j}-1},
$$

whence the argument of the quantity on the left is $-\left(1-1 / n_{j}\right) \arg \left(1-z / \omega_{j}\right)$; since $-\pi / 2<\arg \left(1-z / \omega_{j}\right)<\pi / 2$, the real part of the derivative of $\omega_{j} f_{j}(z)$ is positive throughout the open unit disc, and therefore $\Re F^{\prime}(z)>1$ when $|z|<1$. This implies that $\left|F\left(z_{1}\right)-F\left(z_{2}\right)\right| \geq\left|z_{1}-z_{2}\right|$ for all pairs of points $z_{1}$ and $z_{2}$ in the open unit disc; and because $F(z)$ is continuous in the closed unit disc, it is schlicht in the closed unit disc.

3. The second example. The schlicht function whose Taylor series has Fabry gaps and converges uniformly but not absolutely on $C$ is obtained from the first example by simple modifications. Let

$$
G(z) \equiv z+\sum_{j=1}^{\infty} g_{j}(z),
$$

where

$$
g_{j}(z) \equiv k_{j} z\left\{1-\left[1-\left(z / \omega_{j}\right)^{p_{j}}\right]^{1 / n_{j}}\right\}
$$

the symbols $\omega_{j}$ and $n_{j}$ play the same role as in the first example; $k_{j}$ is a certain real number; and $p_{j}$ is an integer, much smaller than $n_{j}$. For the sake of intuitive clarity, it should be observed that the value of $g_{j}(z)$ is $k_{j} z$ when $\left(z / \omega_{j}\right)^{p j}=1$, 
and that it is small whenever $|z| \leq\left|\omega_{j}\right|$ and $\left(z / \omega_{j}\right)^{p_{j}}$ is very different from one. A rough idea of the image of $C$ under the mapping by $G(z)$ can be obtained by attaching to $C$ a tooth of length $k_{1}$ at each of the points

$$
z=\exp \left[i\left(\phi_{1}+2 \pi h / p_{1}\right)\right], \quad h=0,1,2, \cdots, p_{1}-1,
$$

then adding further sets of teeth as dictated by the parameters $k_{2}, \omega_{2}, p_{2}$, and so forth.

A rigorous proof that the parameters can actually be chosen in such a way that the function $G(z)$ is schlicht and will be schlicht after it has been modified through the introduction of gaps in its Taylor series is based on the study of $\Re \psi^{\prime}(z)$, where

$$
\psi(z) \equiv z\left\{1-\left[1-(z / \omega)^{p}\right]^{1 / n}\right\}
$$

$(|\omega|>1, p$ and $n$ integers, $1 \leq p<n)$. If $t=(z / \omega)^{p}$, then

$$
\psi^{\prime}(z)=1+(1-t)^{1 / n-1}[(1+p / n) t-1] \equiv \Phi(t) .
$$

We wish to show that

$$
\Re \psi^{\prime}(z)>-3 p / n,
$$$$
|z| \leq 1
$$

In order to do this we shall prove that

$$
\Re \Phi(t)>-3 p / n, \quad|t| \leq 1, \quad t \neq 1 .
$$

Since $\Phi(t)$ is holomorphic for $|t| \leq 1, t \neq 1$, it will suffice to show that (12) holds

(a) when $t$ is inside the unit circle (of the $t$-plane) and sufficiently near the point $t=1$;

(b) when $|t|=1, \quad t \neq 1$.

Since the coefficients of the powers of $t$ in the power series of $\Phi(t)$ are all real, we may restrict ourselves in (a) and (b) to values of $t$ whose imaginary part is nonnegative; if $t$ has one of these values,

$$
0 \geq \arg (1-t)>-\pi / 2 \text {. }
$$

(a) Let $t=u+i v$, and consider those values of $t$ for which

$$
u^{2}+v^{2}<1, \quad \frac{1+p / 2 n}{1+p / n}<u<1, \quad 0 \leq v<\frac{p}{2 n^{2}(1+p / n)} .
$$


We then have

$$
0 \leq \arg (1-t)^{1 / n-1}<(\pi / 2)(1-1 / n)
$$

and

$$
0 \leq \arg [(1+p / n) t-1]=\arctan \frac{(1+p / n) v}{(1+p / n) u-1}<\frac{p / 2 n^{2}}{p / 2 n}<\frac{\pi}{2 n},
$$

whence $\Re \Phi(t)>1$.

(b) Let $t=e^{i \theta}$, where $0<\theta \leq \pi$. A simple computation gives

$$
\begin{aligned}
\Re \Phi(t)=1- & \left(2 \sin \frac{\theta}{2}\right)^{1 / n-1} \\
& \times\left\{\left(1+\frac{p}{n}\right) \sin \frac{(n+1) \theta-\pi}{2 n}+\sin \frac{(n-1) \theta+\pi}{2 n}\right\} \\
=1- & \left(2 \sin \frac{\theta}{2}\right)^{1 / n} \cos \frac{\pi-\theta}{2 n} \\
& -\frac{p}{n}\left(2 \sin \frac{\theta}{2}\right)^{1 / n-1} \sin \frac{(n+1) \theta-\pi}{2 n} .
\end{aligned}
$$

$\left(b_{1}\right)$ If $0<\theta \leq \pi /(n+1)$ then, from the second expression for $\Re \Phi(t)$ in (13), we have

$$
\Re \Phi(t) \geq 1-(2 \sin \theta / 2)^{1 / n} \cos [(\pi-\theta) / 2 n]>0 .
$$

$\left(b_{2}\right)$ If $\pi /(n+1)<\theta \leq \pi$, then the content of the braces in the first expression for $\Re \Phi(t)$ in (13) is less than

$$
(1+p / n)(2 \sin \theta / 2) \cos [(\pi-\theta) / 2 n],
$$

and hence

$$
\Re \Phi(t)>1-2^{1 / n}(1+p / n)>-3 p / n .
$$

This establishes the validity of (12), and therefore that of (11).

Now let

$$
\left\{p_{j}\right\}=\{1,2,2,4,4,4,4,8,8,8, \cdots\} ;
$$




$$
\begin{gathered}
k_{j}=1 / p_{j} ; \\
\left\{\phi_{j} / 2 \pi\right\}=\{0,0,1 / 4,0,1 / 16,2 / 16,3 / 16,0,1 / 64,2 / 64, \cdots\} .
\end{gathered}
$$

The choice of the parameters $n_{j}$ and $\rho_{j}$ is similar to the analogous procedure in the first example. However, here we restrict ourselves entirely to the closed unit disc and choose as the region $\Omega_{j}$ the complement, relative to $|z| \leq 1$, of the union of certain neighborhoods of the points

$$
\exp \left[i\left(\phi_{j}+2 \pi h / p_{j}\right)\right], \quad h=0,1,2, \cdots, p_{j}-1 .
$$

These neighborhoods are chosen sufficiently small so that if a point $z$ of the closed unit disc fails to lie in $\Omega_{j}$, it lies in $\Omega_{r}$ whenever $r \neq j$ and $p_{r}=p_{j}$. Furthermore, the indices $n_{j}$ should be greater than $p_{j}$ and such that

$$
\sum_{j=1}^{\infty} 1 / n_{j}<1 / 8 .
$$

In this manner we will again arrive at the result that the series in (10) converges uniformly for $|z| \leq 1$, and that the convergence of the Taylor series for $G(z)$ is not absolute on $|z|=1$ because, as in the first example,

$$
\sum_{M_{j}<m \leq M_{j}+1}\left|a_{m}\right| \geq k_{j}-O\left(2^{-j}\right)
$$

and $\sum_{j=1}^{\infty} k_{j}=\infty$.

The function $G(z)$ has all the properties that are required of the second example (see Summary), except that it fails to possess Fabry gaps. In order to introduce these, we replace each $g_{j}(z)$ by a partial sum $s_{j}(z)$ of its Taylor series. Because the Taylor series of $g_{j}(z)$ and $g_{j}{ }^{\prime}(z)$ converge uniformly in the closed unit disc, it is possible to choose the degrees $P_{j}$ of the polynomials $s_{j}(z)$ large enough so that

$$
\left|g_{j}(z)-s_{j}(z)\right|<2^{-j}
$$

when $|z| \leq 1$ (this ensures uniform convergence of the series

$$
S(z) \equiv z+\sum_{j=1}^{\infty} s_{j}(z)
$$


on the closed unit disc); so that

$$
\left|g_{j}{ }^{\prime}(z)-s_{j}{ }^{\prime}(z)\right|<1 / n_{j}
$$

when $|z| \leq 1$, and in turn

$$
\Re S^{\prime}(z)>1-4 \sum_{j=1}^{\infty} 1 / n_{j}>1 / 2
$$

(this guarantees univalence of the function $S(z)$ in the closed unit disc); and so that the analogue to (14) holds for the Taylor series of $S(z)$. The function $S(z)$ then has the desired properties.

\section{REFERENCES}

1. L. Fejér, La convergence sur son cercle de convergence d'une série de puissance effectuant une représentation conforme du cercle sur le plan simple, C. R. Acad. Sci. Paris 156 (1913), 46-49.

2. G. H. Hardy, A theorem concerning Taylor's series, Quart. J. Pure Appl. Math. 44 (1913), 147-160.

3. F. Herzog and G. Piranian, Sets of convergence of Taylor series I, Duke Math. J. 16 (1949), 529-534.

4. E. Landau, Darstellung und Begründung einiger neuerer Ergebnisse der Funktionentheorie, Second Edition, Springer, Berlin, 1929.

The University of Michigan

Michigan State College 


\title{
EDITORS
}

\author{
HERBERT BUSEMANN \\ University of Southern California \\ Los Angeles 7, California
}

R. M. RoBINSON

University of California

Berkeley 4, California

E. F. BECKENBACH, Managing Editor

University of California

Los Angeles 24, California

ASSOCIATE EDITORS
R. P. DILWORTH
P. R. HALMOS
BQRGE JESSEN
J. J. STOKER
HERBERT FEDERER
HEINZ HOPF
PAUL LÉVY
E. G. STRAUS
MARSHALL HALL
R. D. JAMES
GEORGE PÓLYA
KÔSAKU YOSIDA

\section{SPONSORS}

UNIVERSITY OF BRITISH COLUMBIA

CALIFORNIA INSTITUTE OF TECHNOLOGY UNIVERSITY OF CALIFORNIA, BERKELEY

UNIVERSITY OF CALIFORNIA, DAVIS

UNIVERSITY OF CALIFORNIA, LOS ANGELES

UNIVERSITY OF CALIFORNIA, SANTA BARBARA

OREGON STATE COLLEGE

UNIVERSITY OF OREGON
UNIVERSITY OF SOUTHERN CALIFORNIA STANFORD UNIVERSITY

WASHINGTON STATE COLLEGE

UNIVERSITY OF WASHINGTON

AMERICAN MATHEMATICAL SOCIETY NATIONAL BUREAU OF STANDARDS, INSTITUTE FOR NUMERICAL ANALYSIS

\section{Vari-Type Composition by \\ Cecile Leonard \\ Ruth Stafford}

With the cooperation of

E. F. Beckenbach

E. G. Straus

Printed in the United States of America by

Edwards Brothers, Inc., Ann Arbor, Michigan

UNIVERSITY OF CALIFORNIA PRESS - BERKELEY AND LOS ANGELES COPYRIGHT 1951 BY PACIFIC JOURNAL OF MATHEMATICS 


\section{Pacific Journal of Mathematics}

\section{Vol. 1, No. $1 \quad$ November, 1951}

Ralph Palmer Agnew, Ratio tests for convergence of series............. 1

Richard Arens and James Dugundji, Topologies for function spaces....... 5

B. Arnold, Distributive lattices with a third operation defined ........... 33

R. Bing, Concerning hereditarily indecomposable continua ........... 43

David Dekker, Generalizations of hypergeodesics ............... 53

A. Dvoretzky, A. Wald and J. Wolfowitz, Relations among certain ranges of vector measures.................................... 59

Paul Erdős, F. Herzog and G. Pirani, Schlicht Taylor series whose convergence on the unit circle is uniform but not absolute .......... 75

Whilhelm Fischer, On Dedekind's function $\eta(\tau) \ldots \ldots \ldots \ldots \ldots \ldots . \ldots 3$

Werner Leutert, The heavy sphere supported by a concentrated force ...... 97

Ivan Niven and H. Zuckerman, On the definition of normal numbers ...... 103

L. Paige, Complete mappings of finite groups .................. 111

Otto Szász, On a Tauberian theorem for Abel summability ............. 117

Olga Taussky, Classes of matrices and quadratic fields ............. 127

F. Tricomi and A. Erdélyi, The asymptotic expansion of a ratio of gamma functions .................................... 133

Hassler Whitney, On totally differentiable and smooth functions ......... 143 